\title{
Author Index Vol. 57, 1995
}

Aaby,C. 279 Akanabe,K. 239 Akhtar,S. 48 Ålander,U. 207 Amedee,R.G. 319 Andaz,C. 33

Anniko, M. 60,61,

105,177 Arany, A. 232

Arnold, W. 10,60,61,68,78,82,87 Asami, K. 239 Aust,R. 207

Bachor,E. 129 Bajo-Lorenzana, V. 305 Bartoli,R. 296 Becker, G. 273 Becker, M. 351 Benazzo, M. 39 Benchaou,M. 198 Bende,M. 207 Beppu,R. 269 Berggren, D. 202 Beuth,J. 148

Bircher,AJ. 54 Biurrun, O. 325 Bjerkhoel,A. 202 Boer,M.F. de 165,338 Bonkowsky, V. 22

Carlborg,A. 202 Carlsson-Nordlander, B. 202 Chaudry,M.R. 48 Chen,J. 245 Chhaya,S $\Lambda .286$

Cho, Y.S. 264

Danova, M. 39

Elö,G. 161 Engervall,K. 202 Engström, C.-F. 220 España, R. de 325

Fan,Y. 245 Felix, D. 15 Felix, H. 15 Franchini, G. 39 Freeman, D.J. 256

Gabius,H.-J. 22,343 Gao,Z. 245 Gerlinger, I. 232

Gloddek,B. 10 Gotoh,Y. 122 Graf, P. 328 Grenner, J. 202 Grolman,W. 165,338 Guyot,J.P. 198

Haase, G. 36 Hallén,H. 328 Hanner,P. 202 Harada,Y. 5,100 Hashimoto, K. 135 Hattori,T. 269

Häusler,R. 305,310 Hederstedt, B. 202 Heilbronn,E. 105 Heyworth, T. 33 Higo, R. 333

Hirakawa, K. 5 Hirsbrunner, P. 54 Hirschberg, A. 161 Hisamatu, K. 135 Hofstädter, F. 22, 148, 343 Högmo, A. 202 Holgersson, M. 207 Hosoda, Y. 182 Hultcrantz, M. 1

Igarashi, M. 111,115 Ikezono, T. 122 Imamura, S. 135 Inoue,Y. 94,153 Irifune,M. 210 Ishii,T. 115 Ishimoto,S. 293 Isholt,R.-M. 202 Iurato,S. 141,296 Iwamori, M. 333

Jinnouchi, K. 122 Jong, H.A.A. de 189 Juto,J.-E. 328

Kaga,K. 293 Kaname, H. 115 Kang,M.K. 214 Kano,Y. 224 Kanzaki,J. 94,153, 182

Karmody,C.S. 129 Kato,M. 269 Kawakami, M. 171 Kawashima, K. 210 Kempf,H.-G. 273

Kinoshita, T. 122 Kitamura,M. 269 Knegt,P.P. 165 Koerner, F. 310 Kojima, Y. 224 Kölbeck, K.G. 328 Komatsuzaki, A. 44 Kortschot, H.W. 316 Kristensen, S. 279 Kunihiro, T. 153 Kuno,K. 239 Kuth,G. 36

Laippala, P. 260 Lamprecht, J. 36 Läng,H. 351 Lee,C.H. 289 Lee,K, S. 264 Lenarz, Th. 273 Li,H.-S. 1 Li,J. 245 Lorente, J. 325 Lozupone, E. 296 Lundberg, B. 202

Makimoto, K. 171 Mamikoglu, B. 194 Manabe,Y. 111,250 Mann,WJ. 319 Masuda,M. 182

Matsunaga, T. 153,182 Maurer,J. 319 Meeuwis, C.A. 165,338 Mevio, E. 39 Michaels, L. 228

Min,Y.-G. 214,264,289 Miyawaki,H. 100 Mochizuki, R. 18 Mondain,M. 28 Montandon, P. 198

Morioka,I. 299 Morisaki, H. 239 Murakami, Y. 135

Nakashima, I. 269 Nakashima, T. 239 Nakayama, H. 135 Nathanson, A. 220 Nibu,M. 210

Nielsen, S.M. 279 Nilsson, R. 220 Nishizaki,K. 87,177 Nozawa, I. 135

Occhini,A. 39 Ödkvist,L. 202 Ogata, A. 153 Ogino,S. 210 Ohinata,Y. 171 Okar,I. 194

Onofri,M. 141 Oosterveld, W.J. 189,316 O-Uchi,T. 153

Park,H.M. 214 Pames,L.S. 256 Pawander, R. 122 Petruson,B. 207 Pettersson, A. 105 Phelps, P.D. 228 Prior, AJ. 348 Pröbster,L. 148 Pytel,J. 232

Quaranta, A. 296 
Renard,N. 28 Resta,L. 296 Reuter,G. 299 Ribári,O. 161 Riechelmann, H. 319 Rouiller, E.M. 305 Rowe,S. 33 Ruck, P. 273 Rundcrantz, H. 207 Ryan, A. 28

Safran, A.B. 310 Saito,H. 18,111,250 Saito,T. 111,250 Salama, N.Y. 286 Sato, Y. 153

Schouwenburg, P.F. 165,338 Sehitoglu, M.A. 194 Shin,J.-S. 289 Shinkawa,H. 94 Silvoniemi, P. 260 Sipilä,J. 260 Sobin,J. 220 Sondag, H.N.P.M. 189 Song,B.H. 214,264 Spoelstra, H.A.A. 165 Stenqvist, M. 105 Steuer, M. 22, 343 Steuer,M.K. 22,148,343 Stigbrand,T. 61,68 Strom, A. 68 Strutz,J. 22, 148 Sun,A.-H. 256 Suonpää, J. 260 Suzuki, M. 293 Szabó,G. 161

Takahashi,H. 171 Takumida, M. 5,61,100 Tibbelin,A. 207 Tomiyama, S. 122 Traserra, J. 325 Tschopp, K. 54

Tsunoda, A. 44 Tsuzuki,H. 111

Usami,S.-I. 87,94

Verbeeten, B., Jr. 338 Vibert,D. 310 Vischer, M.W. 305

Walsh, R.M. 286 Weber, B.P. 273 Wildermuth, V. 54

$\mathrm{Xu}, \mathrm{M} .245$

Yagi,T. 18,122 Yamagishi, T. 111 Yamamoto, H. 171 Yamashita, K. 44 Yanagisawa, Y. 44 Yanagita,N. 239,269 Yokoyama, M. 293 Yoshihara, T. 115 Yoshiwara, S. 153 Yun,Y.-S. 264 Zbären,P. 50,351 Zhang, J.S. 305 Zimmermann, A. 50

358

AuthorlndexVol. 57, 1995 